\title{
Measurement and Characterisation of a Diffuse Acoustic Field Using a Phased Array
}

\author{
Jingwei Cheng ${ }^{*}$ (D)
}

\begin{abstract}
A diffuse acoustic field has been increasingly used to infer temporal changes in the structures, such as early dislocations and microcracking. This study explores three different techniques to characterise acoustic field by using a single ultrasonic phased array. The first two techniques are proposed to measure spatial uniformity of wave field by examining differences in the integral of energy and the maximum energy respectively at multiple inspection locations. The third one is developed to evaluate the degree of phase coherence between propagating waves transmitted sequentially by two neighbouring array elements. The efficacy of these techniques are investigated by examining their metrics on simulations and well-known samples. The results suggest that two selected metrics can be used to quantitatively estimate the diffuse field start time as well as the field size by comparing their value with the idealised diffuse state ( $15 \%$ for the energy integral metric, $\eta_{\text {area }}$ and 1 for the phase coherence metric, $\left.\eta_{\text {phase }}\right)$ and identifying the convergence start point.
\end{abstract}

Keywords: Diffuse wave field, Ultrasonic phased array, Sensing, Signal processing

\section{Introduction}

A diffuse acoustic field in which multiply scattered waves propagate is guaranteed to be produced if all the boundaries or heterogeneities within the material volume are diffuse reflectors $[1,2]$. In practice, no boundary acts as perfectly diffuse reflector, however rough and irregular surfaces is assumed to be an adequate approximation if the propagating waves are sensitive to the surface features with the dimensions in the order of wavelength [3, 4]. Furthermore, the recorded diffuse wave is a superposition of all the wave modes from the excitation and the reflections. The type of dominant wave mode in a diffuse field depends on the geometry of the test structure. For example, structural resonances in a diffuse field are principally attributed to Lamb or shear horizontal waves in a thin plate, whereas the response in a thicker structure is dominated by bulk (i.e., shear or longitudinal) waves and surface waves $[5,6]$.

\footnotetext{
*Correspondence: jingwei.cheng@hotmail.com
}

National Safety Engineering Technology Research Centre for Pressure Vessels and Pipelines, Hefei General Machinery Research Institute Co. Ltd., Hefei 230031, China
The identification of a diffuse acoustic field in a specific structure is particularly important for acoustic techniques based on the measurement of diffuse ultrasonic or seismic waves, such as nonlinear ultrasonic diffuse energy imaging (NUI) $[7,8]$ and acoustic emission (AE) testing $[9,10]$ used in structural health monitoring. Those techniques have presented the potential for quantitatively estimating the material degradation and microcracking with high experimental simplicity $[11,12]$. The change in characteristics of wave energy due to the target source can therefore be measured subsequently at any point of the structure in diffuse field $[13,14]$. This is often achieved by employing a small number of sensors or a single phased array to transmit the waves and measure the resultant wave field after multiple reflections throughout the structure [15]. Whilst some previous studies suggest that the essential requirement for the generation of a diffuse field can be assumed to be in the range of 3 to 20 reflections [16, 17], no proof is given on a case-by-case basis. In addition, few quantitative studies, in which the degree of diffuseness is investigated using practical experimental setup has limited the industrial 
applications of the acoustic testing methods, associated with diffuse field measurement [18].

The practical measurement of diffuse waves necessitates a compromise between diffusivity (i.e., the extent to which the field is truly diffuse) and signal-to-noise ratio (SNR) because of two competing effects. First, the acoustic field only slowly tends to the diffuse field state over time [19]. Second, as wave propagation in real structures is dissipative and the acoustic energy is converted to other forms the SNR will decrease with time [20]. Ultimately, compromising values of gate start time $t_{r}$ and window length $T$ for a time window used in the inspection techniques must be found, whereby an adequately diffuse field condition is realised and the amplitude is measurable, i.e., the SNR is sufficient $[21,22]$. From this discussion, it is apparent that the key requirement is to predict the earliest point at which the field can be approximated as a diffuse field state (i.e., the smallest value of $t_{r}$ ).

If an idealised diffuse field is assumed the total wave energy can be uniformly distributed throughout the structure [23, 24]. In addition, the wave components in diffuse field possess random phases $[25,26]$. Therefore, a few methods have been proposed to examine the crosscorrelation or the spatial uniformity of acoustic pressure at different locations [27], whereas lack of comparative study has been conducted systematically on simulations and well-known samples. Furthermore, the highly measurable errors due to reposition of a monolithic transducer have been seen in previous methods. Recent advances in electronic control have however allowed the diffuse field to be measured simultaneously at multiple locations by a single phased array probe [28]. This also potentially improves the practicality of NUI or AE testing by using the same inspection apparatus.

In this paper, three different metrics used for diffuse field verification are proposed and examined on analytical, experimental and numerical data. More specifically, the first two metrics $\left(\eta_{\text {area }}\right.$ and $\left.\eta_{\max }\right)$ evaluate the statistical variation in energy received simultaneously at different location, and the third metric, $\eta_{\text {phase }}$ measures the phase coherence of signals fired independently by two neighbouring transmitters. The analytical model is simulated random noise including the decay coefficients, which is thought to provide a representation of idealised diffuse state. The experiment was designed to measure and predict the formation of a diffuse field in well-known structures. The proposed metrics are then compared in terms of accuracy and limitations. The results demonstrate that the metrics, $\eta_{\text {area }}$ and $\eta_{\text {phase }}$, can be used to identify gate start time $t_{r}$ and window length $T$ by comparing their values with the indicated diffuse state, in which the $\eta_{\text {area }}$ is close to $15 \%$ and the $\eta_{\text {phase }}$ is approximate to 1 .

\section{Methods for Verifying a Diffuse Field}

Previous studies have concluded that the measured envelope area extracted from the signal envelopes can be indicative of the signal amplitude such that estimating whether a field is diffuse is then simply a case of mapping the variation of the envelop area across the surface of the structure [3]. However, the technique was limited to measurement errors from repositioning the receiver because simultaneous sampling at a large number of positions were not realisable. The phased array system that consists of multiple electronically controlled channels potentially recently enabled the whole process to be completed without those limitations. Hence, it is advantageous to exploit its capability on diffuse field verification and ultimately integrate the measurement of diffuse field with those relevant nondestructive testing (NDT) techniques using a phased array.

The acoustic source used in this work is assumed as a 64-element ultrasonic phased array with nominal centre frequency at $5 \mathrm{MHz}$. The full matrix capture (hereinafter referred to as FMC) [29] is implemented to fully use the array elements at as many locations as possible so that more diffusivity relative to the source locations can be taken into account. Although works [3] reported that the envelope area measurement was favoured over maximum amplitude both will be examined more comprehensively and compared here. In addition, it is thought to evaluate spatial variations in energy rather than amplitude, which is attributed to the definition of a diffuse field. Last but not least, the energy area by calculating the integral of energy over the window (as illustrated in Figure 1) and the maximum energy in the window are used in the first two metrics $\left(\eta_{\text {area }}\right.$ and $\left.\eta_{\max }\right)$ respectively. Assuming $f_{n, m}(t)$ are the FMC time-domain received signals for each combination of transmit $(n)$ and receive $(m)$ elements in sizes of $N_{n}$

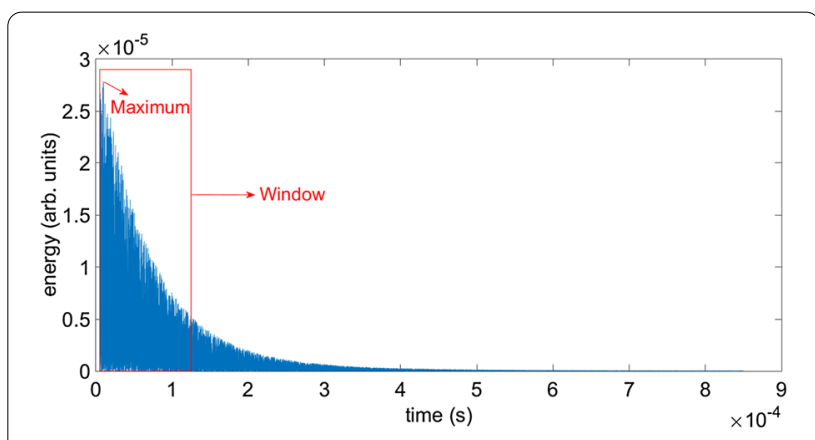

Figure 1 An example time trace of simulated incoherent noise for schematically illustrating the selection of windowed energy (with a window size, $T$ ) and the maximum energy in arbitrary units 
and $N_{m}$ respectively the metric for evaluating variations in energy area of windowed signals, $\eta_{\text {area }}$, can be expressed as:

$$
\eta_{\text {area }}=100 \sum_{n=1}^{N_{n}} \frac{\sigma\left(\int_{t_{r}}^{t_{r}+T}\left|f_{n, 1 \rightarrow N_{m}}(t)\right|^{2} \mathrm{~d} t\right)}{N_{n} \overline{\int_{t_{r}}^{t_{r}+T}\left|f_{n, 1 \rightarrow N_{m}}(t)\right|^{2} \mathrm{~d} t}} \%,
$$

where $\sigma$ denotes the standard deviation. Further, given that the maximum amplitude in each window of FMC signals is denoted as $f_{n, m}^{\max }$ the metric for evaluating variations in maximum energy, $\eta_{\max }$ can be expressed as

$$
\eta_{\max }=100 \sum_{n=1}^{N_{n}} \frac{\sigma\left(\left|f_{n, 1 \rightarrow N_{m}}^{\max }\right|^{2}\right)}{N_{n} \overline{\left|f_{n, 1 \rightarrow N_{m}}\right|^{\max }}} \% .
$$

Here, the above metrics were implemented to measure the normalised standard deviations of energy integrals and maximum energy respectively from the same time traces received by 64 elements. Note that the selection of transmitters will be discussed later and the window length, $T$, was thought to be $0.12 \mathrm{~ms}$, the empirical value used in NUI as stated in $[8,11]$. Finally, their averaged value (termed $\eta_{\text {area }}$ and $\eta_{\max }$ ) were plotted against gate start time, $t_{p}$ in order to represent the level of diffusivity with corresponding wave energy at different times. Previous experimental results state that the initial reflected signals account for most of the variation and it necessitates a delay to allow the energy to be evenly distributed $[3,24]$. Therefore, these two metrics should be expected to converge to a lower value with increasing $t_{r}$.

The other method was developed by considering the change in phase coherence evolved from coherent field to diffuse field. In diffuse field, all the signals could be expected to behave like white noise (i.e., the waves at different locations are uncorrelated to each other) [3]. Furthermore, selection of the most representative wave sources to examine their relative phase coherence significantly determines the effectiveness of this method. This is because two neighbouring transmitters in an array are preferred over two transmitters far away from each other in terms of the similarity of their received signals in the same time window in a coherently scattered field. Therefore, the summation of their windowed signals fired independently by two neighbouring transmitters and received by all the elements in an array is approximate to twice of the either one in a coherently reflected field. However, the distribution of the reflected wave field is independent of the incident angle (i.e., random in amplitude and direction) in real structures, whereby the boundaries and the grains are diffuse reflectors. Hence, a diffuse field will be generated over time and the arrival of diffuse field can be indicated by evaluating the averaged change in phase coherence between propagating waves transmitted by all the combinations of two neighbouring elements and received by all the elements in a phased array.

Once the propagating waves from two neighbouring sources become uncorrelated in diffuse field, their summation is approximate to $\sqrt{2}$ times of each signals which can be attributed to random waveform combinations $(=\sqrt{n} A)$ where $n$ denotes number of component waveforms and $A$ refers to amplitude of component waveform). A compensation factor of 2 is applied to the individual waveform, such that the ratio of the individual amplified waveform to the resultant waveform in energy should be in a range of from 0.5 to 1 (suggest an evolution from coherent wave field to diffuse wave field). The metric is finally averaged from all the pairs of neighbouring transmission elements. As a consequence of calculating the windowed energy extracted from the same signals used in estimating $\eta_{\text {area }}$ and $\eta_{\text {max }}$, the metric for evaluating the degree of their phase coherence, $\eta_{\text {phase }}$ may be written as follows:

$$
\eta_{\text {phase }}=\sum_{n=1}^{N_{n}} \frac{2 \sum_{m=1}^{N_{m}} \int_{t_{r}}^{t_{r}+T}\left|f_{n, m}(t)\right|^{2} \mathrm{~d} t}{N_{n} \sum_{m=1}^{N_{m}}\left(\int_{t_{r}}^{t_{r}+T}\left|f_{n, m}(t)+f_{n \pm 1, m}(t)\right|^{2} \mathrm{~d} t\right)} .
$$

\section{Experimental Generation of an Evolution from Coherent Field to Diffuse Field}

The 64-element array probe (Imasonic, France) with nominal centre frequency of $5 \mathrm{MHz}$ and pitch of 0.6 $\mathrm{mm}$ controlled by a commercial 128-channel controller (Micropulse FMC, Peak NDT, UK) was positioned at the centre of top surface (as illustrated in Figure 2). The two specimens used in this study (as presented in Figure 2(a) and (b)) are made of aluminium ( $\mathrm{Al} 2014$ ) and mild steel (ASTM A36) with the same dimensions of the ones used in NUI works $[8,11]$. The FMC data used for subsequent analysis were produced by sequentially firing each array element and capturing the signals by all the array elements simultaneously.

As the incoherent noise has a significant effect on decayed signals in diffuse field and its contribution increases with time the reception gain should be altered to ensure the received amplitude from each set of captured data is always at the equivalently high level. The window length, $T$, for each FMC was set as $0.06 \mathrm{~ms}$ (half of the standard window length used in previous works $[8,11])$, so as to frequently select the optimum reception gain corresponding to different gate start time, $t_{r}$. Further, the initial gate start time was chosen to $0.005 \mathrm{~ms}$ after which the crosstalk was no longer present and the first 


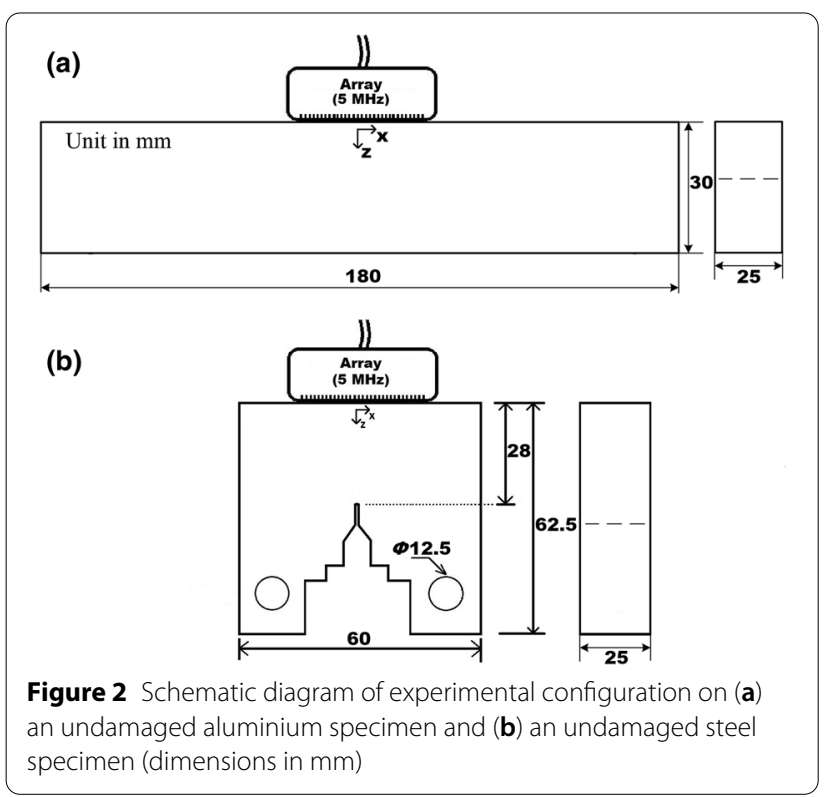

Table 1 Gate start time, reception gain and maximum amplitude corresponding to twelve sets of FMC time traces on the aluminium sample

\begin{tabular}{lll}
\hline Gate start time $\boldsymbol{t}_{\boldsymbol{r}}(\mathbf{m s})$ & Reception gain (dB) & $\begin{array}{l}\text { Maximum received } \\
\text { amplitude (arb. units) }\end{array}$ \\
\hline 0.005 & 23 & 0.991 \\
0.03 & 35 & 0.997 \\
0.09 & 46 & 0.986 \\
0.15 & 49 & 0.969 \\
0.21 & 55 & 0.990 \\
0.27 & 55 & 0.995 \\
0.33 & 59 & 0.996 \\
0.39 & 61 & 0.993 \\
0.45 & 64 & 0.945 \\
0.51 & 66 & 0.959 \\
0.57 & 67 & 0.973 \\
0.63 & 68 & 0.920 \\
0.69 & 70 & 0.995 \\
\hline
\end{tabular}

coherent reflections from boundaries start to emerge. It should be noted that the reception gains (used for maintaining the same contribution of incoherent noise) were determined by finding the maximum received amplitude in each window close to 1 (the saturated value of the array controller). For example, twelve sets of FMC time traces were acquired sequentially on the aluminium sample with the parameters listed in Table 1 and all the signals were normalised subsequently to $23 \mathrm{~dB}$, used in the first capture. From Table 1, the selected reception gain at which the received amplitude nearly approaches the hardware limit (i.e., 1) usually increases with gate start time, thereby suppressing the effect of incoherent noise. Note that the last set was indicated by capturing with the highest instrumental reception gain, $70 \mathrm{~dB}$. Consequently, all the time traces stitched together to form one resulting set of FMC time traces from $0.005 \mathrm{~ms}$ to 0.85 ms.

\section{Analytical Generation of a Diffuse Field}

Since the behaviour of propagating waves in perfectly diffuse state is random, similar to that of white noise it is worth examining the proposed metrics on simulated random signals. This should deliver a benchmark for an idealised diffuse field, such that the evaluated experimental and numerical results can be better appreciated.

As illustrated in Figure 3(a), the white noise, termed $f_{n, m}^{W}(t)$, can be simulated by generating uniformly distributed random variables within specified interval (from -0.4 to 0.4 ) using a standard programming language (MALTLAB R2016a) because incoherent signal is a zero-mean random process. In addition, the decay rate was extracted by fitting a two-term exponential model $\left(y_{n, m}(t)=o_{1} e^{b t}+o_{2} e^{d t}\right.$, where $o_{1}$ and $o_{2}$ are amplitude factors, $b$ and $d$ are decay coefficients) to the stitched experimental FMC data set (an example time trace is displayed in Figure 3(b)). These coefficients were calculated by using the 'Fit' function and specifying its type as 'exp2' in MATLAB software. Note that the two-term exponential model rather than a one-term model was selected due to better approximation of multi-mode wave decay. The simulated random signals in the same size of FMC data were multiplied by the corresponding exponential fits, $y_{n, m}(t)$ to obtain the decayed white noise, denoted as $\hat{f}_{n, m}^{W}(t)$, such that the effect of attenuation in wave propagation on the proposed metrics can be quantified.

\section{Methodology to Predict a Diffuse Field Using Proposed Metrics}

The data obtained from experiments and analytical simulations are now analysed by three proposed metrics, thereby delivering a systematic methodology for diffuse field prediction on any structure. In most of the ultrasonic applications, the fundamental frequency is examined hence the wave propagation at the fundamental frequency in diffuse state is critical to these techniques. As a consequence, the metrics should assess the diffusivity of wave components particularly at the fundamental frequencies, which can be accomplished by introducing a gaussian windowed filter in post-processing.

Specifically, the experimental FMC signals, $f_{n, m}(t)$, the decayed white noise, $\hat{f}_{n, m}^{W}(t)$ and the white noise, $f_{n, m}^{W}(t)$ were selectively processed by a gaussian windowed filter and then examined by three different metrics $\left(\eta_{\text {area }}\right.$, 

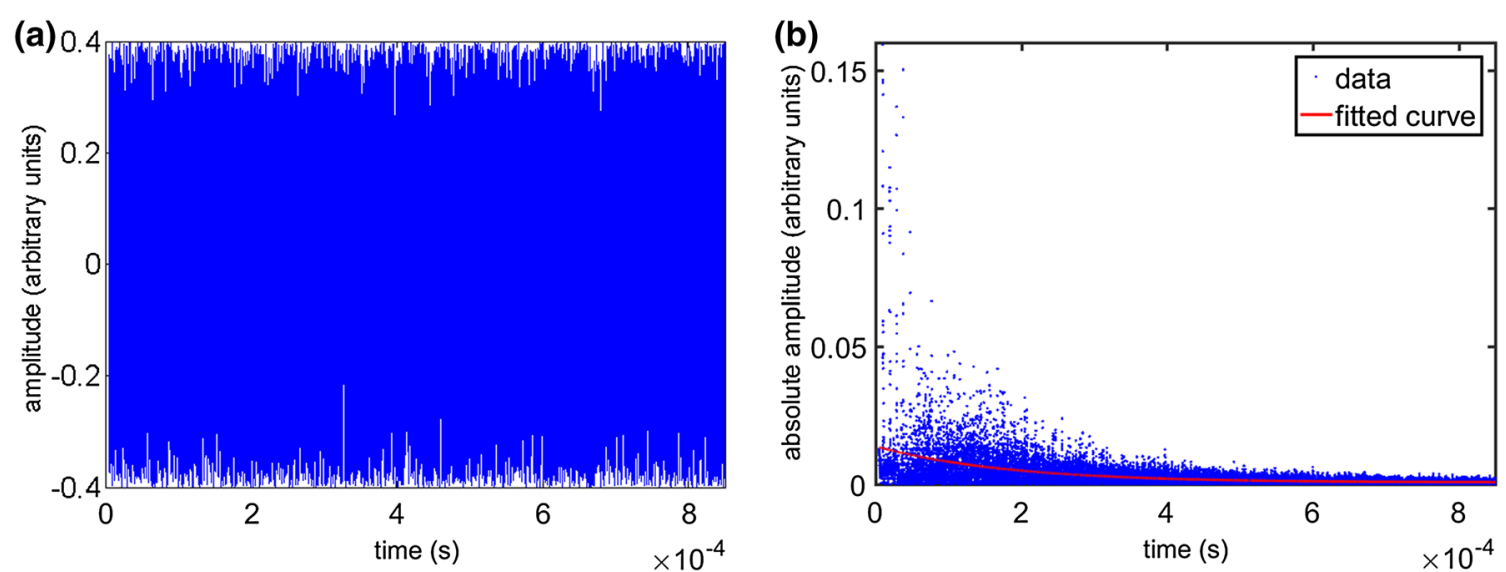

Figure 3 a Simulated white noise, $f_{n, m}^{W}(t)$ against time and $(\mathbf{b})$ an example time trace including the exponential fit

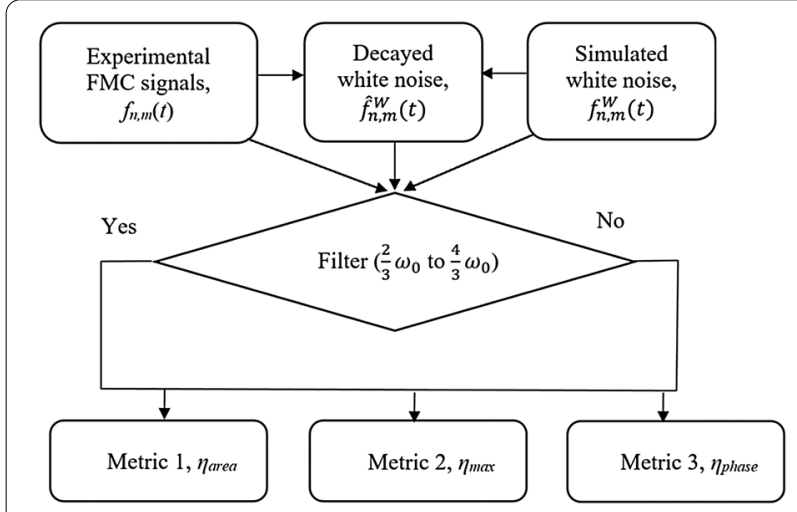

Figure 4 Flow chart of methodology for diffuse field verification

$\eta_{\max }$ and $\eta_{\text {phase }}$ ), in order to predict a diffuse field (as illustrated in Figure 4). Note that $f_{n, m}(t), \hat{f}_{n, m}^{W}(t)$ and $f_{n, m}^{W}(t)$ are input to the identical filter independently. The metrics were plotted against the gate start time, $t_{r}$, from $0.005 \mathrm{~ms}$ to $0.85 \mathrm{~ms}$ (this range was indicated by limits of hardware reception gain). In addition, the window length, $T$, was initially selected as $0.12 \mathrm{~ms}$ as a consequence of the standard parameter used in NUI. Therefore, the outcome of this process will help determine the earliest approach of the diffuse field as well as understand the underlying physics behind the formation of diffuse field in real structures.

First, the relationship between metric, $\eta_{\text {area }}$ and gate start time, $t_{r}$, on the aluminium sample is displayed in Figure 5. This figure indicates the variations in windowed energy, which were post-processed from experimental FMC signals and white noise. A gaussian windowed filter from $2 \omega_{0} / 3$ to $4 \omega_{0} / 3$ was selectively implemented to those data prior to evaluations through proposed metrics. The results from simulated white noise in Figure 5

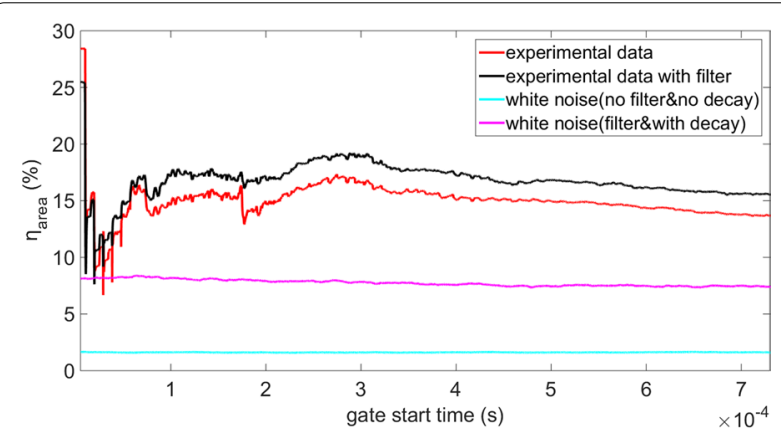

Figure 5 Diffuse field verification on white noise and experimental data using metric $\eta_{\text {area }}$ against gate start time

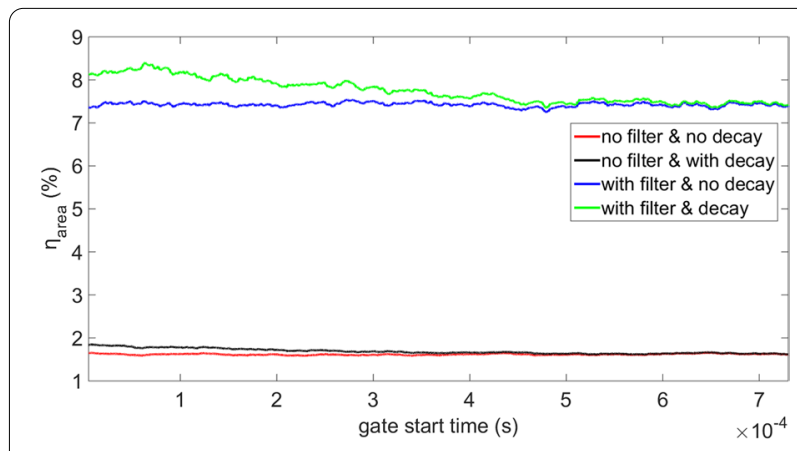

Figure 6 Diffuse field verification on white noise with or without filter and decay using metric $\eta_{\text {area }}$

indicate the benchmarks for an idealised diffuse field. Most importantly, the effect of exponential decay extracted from experimental data and the gaussian windowed filter has measurable contributions to the metric, $\eta_{\text {area }}$. Their corresponding influence can be observed in Figure 6, which demonstrates that the incoherent signals 
within a narrow band tend to have much higher value in $\eta_{\text {area }}$ (i.e., less diffusivity), and the metric on decayed signals is likely to inherit the characteristics of their decay rate.

The metric, $\eta_{\text {area }}$, on experimental FMC signals in Figure 5 suggest a good agreement with previously observed variation of the amplitude envelope measurements about $15 \%$ [3]. Specifically, the experimental results using the metric $\eta_{\text {area }}$ suggest that a diffuse wave field occurs at $0.1 \mathrm{~ms}$ by observing the converged start point and the filtered data provide the higher value in $\eta_{\text {area }}$ within predicted diffuse field, which is consistent to the observation from white noise. Most importantly, this predicted gate start time for a diffuse field is same as the empirical one used in NUI experiments [8] that delivers good NUI performance on crack detection.

The metric $\left(\eta_{\max }\right)$ indicating the variation in maximum energy is plotted in Figure 7 with respect to gate start time, $t_{r}$. Its results are very similar to those in Figure 5 , but the overall variations increase by 20 percent and small fluctuations with increasing $t_{r}$ due to too small number of samples in a statistical problem (i.e., when calculating the standard deviations of overall energy in a wave field). This fact will be confirmed later by the window size study (a converged relationship between $\eta_{\text {area }}$ and window length $T$ as presented in Figure 9) Therefore, the metric $\left(\eta_{\text {area }}\right)$ is favoured over the metric $\left(\eta_{\max }\right)$ as a consequence of the most representative measurement in overall wave field energy.

The change in the metric $\left(\eta_{\text {phase }}\right)$, by which the extent of phase coherence between signals fired by two neighbouring transmitters is reflected, with increasing $t_{r}$ is displayed in Figure 8. As mentioned before, the expected range in $\eta_{\text {phase }}$, indicating the change from coherent field to diffuse field, is from 0.5 to 1 . As a consequence of a phase-dependent method, the filter and the attenuation rate barely have influence on white noise, which can be reflected by the overlapped curves (in cyan and magenta

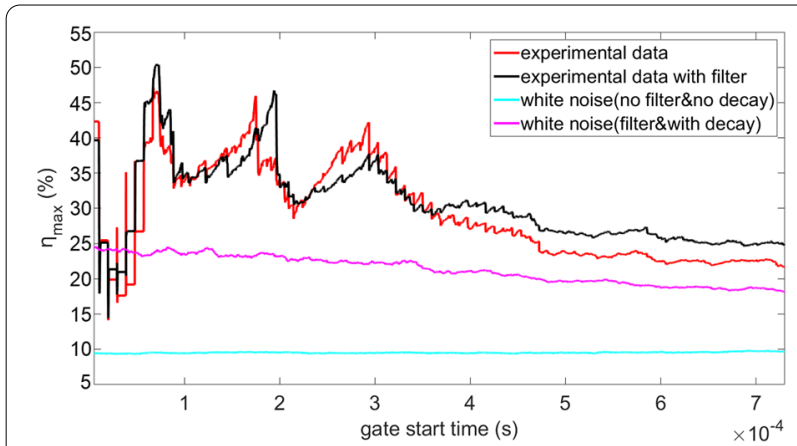

Figure 7 Diffuse field verification on white noise and experimental data using metric $\eta_{\max }$ against gate start time

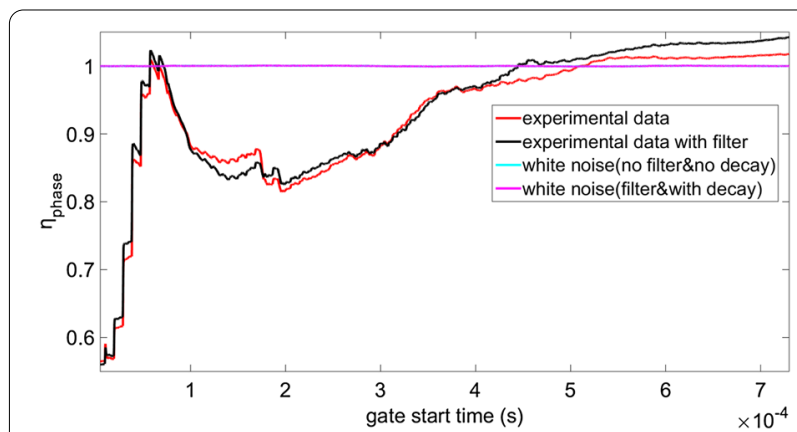

Figure 8 Diffuse field verification on white noise and experimental data using metric $\eta_{\text {phase }}$

colours) in Figure 8. Furthermore, the metric ( $\left.\eta_{\text {phase }}\right)$ on white noise (uncorrelated signals) is consistently with a value of 1 as expected. For the experimental data, the filter also has little effect on this metric. The diffuse state might be indicated from $t_{r}$ at $0.07 \mathrm{~ms}$ due to the corresponding $\eta_{\text {phase }}$ value at 1 although the metric then decreases by approximately 0.15 and then converges to the value around 1.02. This small decrease is possibly because the surface waves not only attenuate more slowly than the bulk waves [30], but also become diffuse much later due to high aspect ratio of this structure (6:1). That is to say, the bulk waves initially at higher intensity will reach diffuse state earlier due to significantly more reflections from two horizontal boundaries closer to each other in z-direction (as illustrated in Figure 2). This fact will be further confirmed by examining $\eta_{\text {phase }}$ on a specimen with lower aspect ratio (1.04:1), as presented in Figure 12 . The metric, $\eta_{\text {phase }}$ evaluating experimental data have the small decrease prior to the previously predicted diffuse field start time $(0.1 \mathrm{~ms})$, which might be attributed to the existing surface wave components in high intensity before $0.1 \mathrm{~ms}$ in Figures 3 and 7.

The window length, $T$, is studied by plotting the metrics $\left(\eta_{\text {area }}\right.$ and $\left.\eta_{\text {phase }}\right)$ against $t_{r}$, because it is important

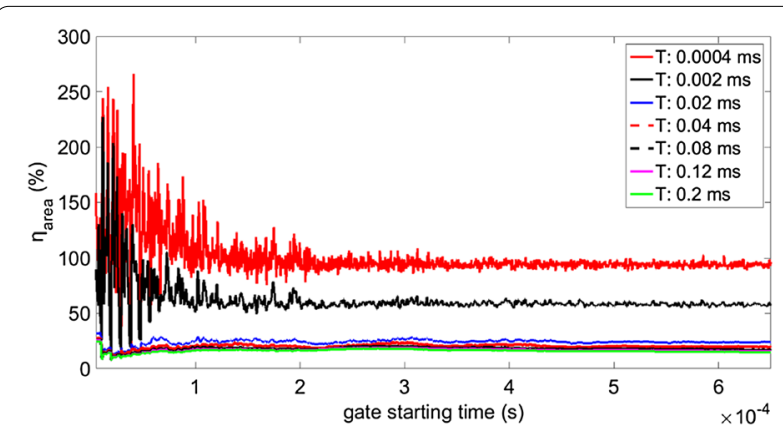

Figure 9 Diffuse field verification on experimental data with different window size using metric $\eta_{\text {area }}$ 
to select the most representative samples in a predicted diffuse field with the smallest window required. In theory, the smaller number of sample (e.g., the single sample used in the metric $\eta_{\max }$ ) are more likely to provide the large error when measuring standard deviations of the statistical energy in diffuse wave field. However, there should be an upper limit for the size of sample to be estimated, which delivers a saturated measurement of diffusivity.

Therefore, the size of window length, $T$, was varied from $0.0004 \mathrm{~ms}$ to $0.2 \mathrm{~ms}$. As presented in Figure 9, the metric, $\eta_{\text {area }}$, was first plotted against $t_{r}$, in order to explore the effect of window size on variation between windowed energy received at different location. It clearly demonstrates that the relationship between the size of $T$ and $\eta_{\text {area }}$ is exponentially inverse. In particular, the $\eta_{\text {area }}$ converges with increasing size of $T$ from the empirical $T$ with $0.12 \mathrm{~ms}$ (used in NUI imaging and previous studies) by observing the overlapped curves between $T$ with $0.12 \mathrm{~ms}$ and $0.2 \mathrm{~ms}$ in Figure 9. It should be also noted that the small size of $T$ (e.g., $0.0004 \mathrm{~ms}$ and $0.002 \mathrm{~ms}$ ) contributed to more fluctuations and larger offset. As a consequence of the results, the size of $T$ with $0.12 \mathrm{~ms}$ is suggested to be used.

Similarly, the same window length study was performed by examining the metric, $\eta_{\text {phase }}$ with increasing gate start time, $t_{r}$. The results in Figure 10 suggest that the larger window size delivers more rapidly converged value in the idealised diffuse state with increasing $t_{r}$. In addition, the metric, $\eta_{\text {phase }}$ becomes converged at $T$ of $0.12 \mathrm{~ms}$ with increasing window size and the three neighbouring pairs was able to provide the same prediction of diffuse field as the entire array transmitters.

Furthermore, the robustness of these two metrics is examined on the same type of steel CT specimen used in experiments [11] for performing NUI technique on crack monitoring, whereby the size of $T$ was used as $0.12 \mathrm{~ms}$ and the resulting time traces from $0.005 \mathrm{~ms}$

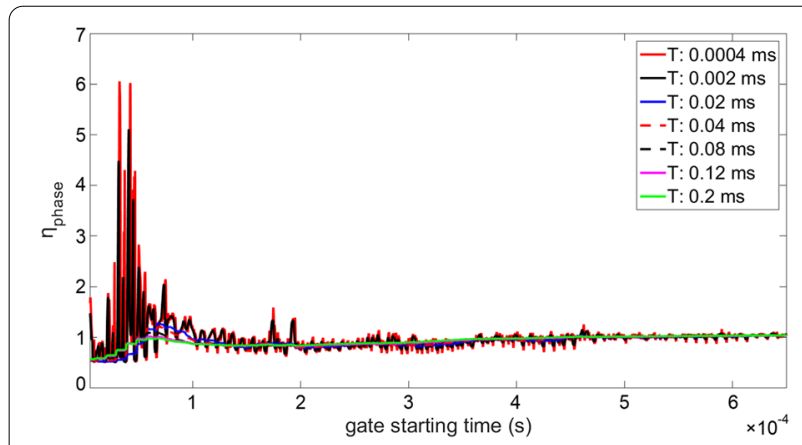

Figure 10 Diffuse field verification on experimental data with different window size using metric $\eta_{\text {phase }}$ to $0.4 \mathrm{~ms}$ was combined from nine sets of FMC data were acquired independently with different gains (from $36 \mathrm{~dB}$ to $70 \mathrm{~dB}$ ). Note that the white noise here is also produced from combining simulated random signal and exponential decay rate extracted from the experimental resulting time traces. The results in Figure $11 \mathrm{dem}-$ onstrate that the metric $\eta_{\text {area }}$ on filtered experimental data implies good agreement with the empirical values used in NUI works [11] ( $T$ of $0.12 \mathrm{~ms}$ with the gate start time, $t_{r}$, at $0.1 \mathrm{~ms}$ ) and that of filtered white noise by observing the converged start point at approximately $0.07 \mathrm{~ms}$ (where $\eta_{\text {area }}$ is around $15 \%$ as the indicated diffuse state). In addition, the metric $\eta_{\text {phase }}$ as presented in Figure 12 identifies the convergence to 0.95 (very close to the idealised diffuse state, 1 ) from around $0.07 \mathrm{~ms}$ in good agreement. It should be noted that the surface of this steel sample in contact with the probe has onethird length of the aluminium one, so that the state of surface waves is expected to become diffuse three times faster due to more reflections from boundaries. As a consequence, the small decrease observed after the predicted diffuse start time $(0.07 \mathrm{~ms})$ in Figure 8 is probably attributed to the surface waves as discussed before.

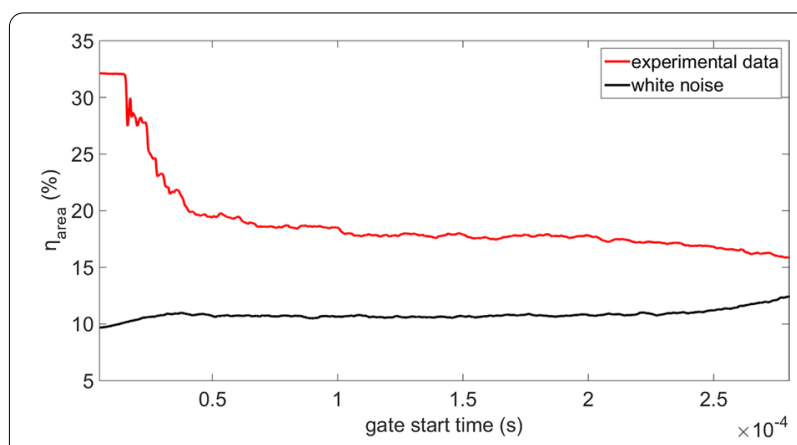

Figure 11 Diffuse field verification on white noise and experimental data using metric $\eta_{\text {area }}$

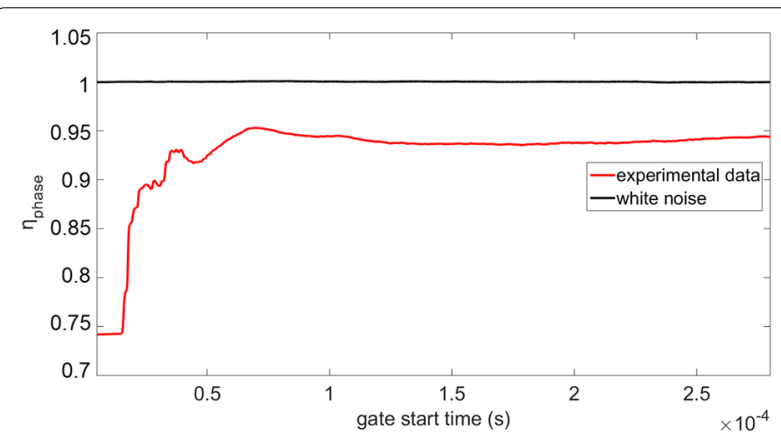

Figure 12 Diffuse field verification on white noise and experimental data using metric $\eta_{\text {phase }}$ 


\section{Conclusions}

The results from the aluminium specimen suggest that both of the metrics $\eta_{\text {area }}$ and $\eta_{\text {phase }}$ were able to effectively identify the change from coherent field to diffuse field of experimental signals according to the reference values generated from decayed white noise. Note that the effect of both the exponential decay extracted from experimental data and the gaussian windowed filter has been found to provide considerable contributions to the metrics. Consequently, the simulated white noise including both of them delivers the best approximation to the experimental data in terms of diffusivity indicated by the proposed metrics. Furthermore, the effect of window size, $T$, was studied by using the same metrics $\eta_{\text {area }}$ and $\eta_{\text {phase }}$. Its results demonstrated that the larger $T$ delivered higher diffusivity and the preferred size of $0.12 \mathrm{~ms}$ indicated by the converged values of $\eta_{\text {area }}$ and $\eta_{\text {phase }}$ was consistent to the empirical value obtained from the experimental NUI performance on crack detection. Investigations using these two metrics were conducted on the same type of steel CT specimens used in previous NUI experiments [11]. The consistent results corresponding to empirical values were confirmed by observing the $t_{r}$, at which both $\eta_{\text {area }}$ and $\eta_{\text {phase }}$ converged to reference values of diffuse state.

In summary, both of the metrics $\eta_{\text {area }}$ and $\eta_{\text {phase }}$ on examining $t_{r}$ and $T$ demonstrated good agreements with their empirical values from the experimental NUI performance on crack detections of the same structures (one aluminium CT specimen and one steel CT specimen), hence, they are thought to be able to quantitatively predict the diffuse field start time as well as the optimum window size $T$ by comparing their value with the idealised diffuse state ( $15 \%$ for $\eta_{\text {area }}$ and 1 for $\eta_{\text {phase }}$ ) and identifying the convergence start point. It should be noted that in some cases the surface waves might lead to errors in judging the diffuse state, particularly with excitation on a long surface of a structure. For the specimens used in this study, their uniform materials have given rise to less reflections and scatterings from heterogeneities, whereas the practical structures with similar geometries containing anisotropic materials should be prone to becoming diffuse at an earlier stage. Consequently, the proposed prediction methods using the metrics, $\eta_{\text {area }}$ and $\eta_{\text {phase }}$, can be implemented to facilitate the practical measurements using NUI method or AE technique in application to non-destructive testing and structural health monitoring.

\section{Acknowledgements}

The author would like to thank B. Drinkwater and J. Potter for their assistance with some advice on methods.
Authors' Contributions

JC designed research, performed research, analysed data, and wrote the paper. The author read and approved the final manuscript.

\section{Authors' Information}

Jingwei Cheng is currently a senior engineer at National Safety Engineering Technology Research Centre for Pressure Vessels and Pipelines, Hefei General Machinery Research Institute Co. Ltd., China. He received his B.Eng. and Ph.D. degrees in Mechanical Engineering from University of Bristol, UK, in 2014 and 2018, respectively. His research interests mainly include phased array testing and nonlinear ultrasonics.

\section{Funding}

Supported by Anhui Provincial Natural Science Foundation of China (Grant No. 2008085J24), Anhui Provincial Science and Technology Major Project of China (Grant No. 201903a05020010), Young Talent Support Program of China Association for Science and Technology (Grant No. [2020] No.87), Doctoral Science and Technology Foundation of Hefei General Machinery Research Institute (Grant No. 2019010381), and Anhui Provincial Key Research and Development Plan of China (Grant No. 202004a05020003).

\section{Competing Interests}

The author declares no competing financial interests.

Received: 26 October 2020 Revised: 2 July 2021 Accepted: 15 November 2021

Published online: 04 December 2021

\section{References}

[1] R S Langley. On the diffuse field reciprocity relationship and vibrational energy variance in a random subsystem at high frequencies. J. Acout. Soc. Am., 2007, 121: 913-921.

[2] CVan, E Reynders. Gaussian orthogonal ensemble modeling of built-up systems containing general diffuse components and parametric uncertainty. Journal of Sound and Vibration, 2021, 501:116045.

[3] M J Evans, P Cawley. Measurement and prediction of diffuse fields in structures. J. Acoust. Soc. Am., 1999, 106: 3348-3361.

[4] THayashi. Defect imaging for plate-like structures using diffuse field. J. Acoust. Soc. Am., 2018, 143(4): EL260-65.

[5] M J Evans, J R Webster, P Cawley. Design of a self-calibrating simulated acoustic emission source. Ultrasonics, 2000, 37(8): 589-594.

[6] S Nakao, T Hayashi. Non-contact imaging for delamination using diffuse field concept. Jpn. J. Appl. Phys., 2019, 58: SGGB07.

[7] G Bunget, J Rogers, C J Bunget, et al. Imaging fatigue damage precursors based on nonlinear phased array ultrasonic measurements of diffuse field. ASME J. Nondestructive Evaluation, 2021, 4(1): 011004.

[8] A J Croxford, J Cheng, J N Potter. Nonlinear phased array imaging. SPIE Conference on Health Monitoring of Structural and Biological Systems, Las Vegas, USA, March 20-24, 2016: $98052 B$.

[9] R Snieder, A Gret, H Doumaand, et al. Coda wave interferometry for estimating nonlinear behavior in seismic velocity. Science, 2002, 295 : 2253-2255.

[10] B S Wu, G C McLaskey. Broadband calibration of acoustic emission and ultrasonic sensors from generalized ray theory and finite element models. J. Nondestruct. Eval., 2018, 37(1): 1-16.

[11] J Cheng, J N Potter, A J Croxford, et al. Monitoring fatigue crack growth using nonlinear ultrasonic phased array imaging. Smart Mater. Struct., 2017, 26: 055006

[12] L Griffiths, O Lengliné, M J Heap, et al. Thermal cracking in Westerly Granite monitored using direct wave velocity, coda wave interferometry, and acoustic emissions. Journal of Geophysical Research: Solid Earth, 2018, 123(3): 2246-2261.

[13] Y Zhang, VTournat, O Abraham, et al. Nonlinear coda wave interferometry for the global evaluation of damage levels in complex solids. Ultrasonics, 2017, 73: 245-252. 
[14] J E Michaels, T E Michaels. Detection of structural damage from the local temporal coherence of diffuse ultrasonic signals. IEEE Trans. Ultrason. Ferroelectr. Freq. Control, 2005, 52: 1769-1782.

[15] H Zhang, Y Liu, G Fan, et al. Sparse-TFM imaging of Lamb waves for the near-distance defects in plate-like structures. Metals, 2019, 9(5): 503.

[16] D M Egle. Diffuse wave fields in solid media. J. Acout. Soc. Am., 1981, 70: 476-480.

[17] R B Clough. A scalar approach to acoustic emission. Proc. International Symposium on Vibroacoustic Characterisation of Materials and Structures, USA, 1992: 1-6,

[18] F Martellotta. Do we still need diffuse field theory? J. Acoust. Soc. Am., 2018, 144(3): 1951.

[19] XWang, J Chakraborty, A Bassil, et al. Detection of multiple cracks in fourpoint bending tests using the coda wave interferometry method. Sensors, 2020, 20(7): 1986.

[20] T McKenzie, DT Murphy, G Kearney. Diffuse-field equalisation of binaural ambisonic rendering. Applied Sciences, 2018, 8(10): 1956.

[21] H Zhang, H Zhang, J Zhang, et al. Wavenumber imaging of near-surface defects in rails using green's function reconstruction of ultrasonic diffuse fields. Sensors, 2019, 19(17): 3744.

[22] C Hei, M Luo, P Gong, et al. Quantitative evaluation of bolt connection using a single piezoceramic transducer and ultrasonic coda wave energy with the consideration of the piezoceramic aging effect. Smart Materials and Structures, 2020, 29(2): 027001

[23] R L Weaver. Diffuse waves in finite plates. J. Acoust. Soc. Am., 1984, 94: 319-335.

[24] R L Weaver. Diffuse elastic waves at a free surface. J. Acoust. Soc. Am., 1985, 78: 131-136.

[25] M Tamart, F Sarrazin, E Richalot, et al. Diffuse field cross-correlation in a reverberantion chamber. 2020 IEEE International Symposium on Antennas and Propagation and North American Radio Science Meeting, Montreal, Canada, 2020: 1179-1180.

[26] M Davy, J R De, P Besnier. Green's function retrieval with absorbing probes in reverberating cavities. Physical Review Letters, 2016, 116(21): 213902.

[27] W Aquino, J Rouse. Optimization approach for designing diffuse acoustic fields on demand. J. Acout. Soc. Am., 2020, 148(4): 2605.

[28] X Liu, J Ma, HWang, et al. Ultrasonic scattered field distribution of one and two cylindrical solids with phased array technique. Chin. J. Mech. Eng., 2019, 32:96

[29] C Holmes, B W Drinkwater, P D Wilcox. Post-processing of the full matrix of ultrasonic transmit-receive array data for non-destructive evaluation. NDT\&E Int., 2005, 38: 701-711.

[30] K Uenishi. Rupture, waves and earthquakes. Proc. Jpn. Acad. Ser. B Phys. Biol. Sci., 2017, 93: 28-49.

\section{Submit your manuscript to a SpringerOpen ${ }^{\circ}$ journal and benefit from:}

- Convenient online submission

- Rigorous peer review

- Open access: articles freely available online

- High visibility within the field

- Retaining the copyright to your article

Submit your next manuscript at $\gg$ springeropen.com 